\title{
Xylose-glucose co-fermentation to ethanol by Escherichia coli strain MS04 using single- and two-stage continuous cultures under micro-aerated conditions
}

\author{
Marco T. Fernández-Sandoval', Juvencio Galíndez-Mayer², Francisco Bolívar' ${ }^{1}$, Guillermo Gosset ${ }^{1}$, \\ Octavio T. Ramírez ${ }^{3}$ and Alfredo Martinez ${ }^{1 *}$ (1)
}

\begin{abstract}
Background: Simultaneous co-fermentation of mixed sugars is an important feature to consider in the production of ethanol from lignocellulosic biomass hydrolysates because it enhances the overall ethanol yield and volumetric productivity during fermentation. Continuous cultures can be used during ethanol production from lignocellulosic hydrolysates to prevent catabolite repression by glucose on other sugars, such as xylose, and thus promote the simultaneous and total consumption of sugars and reduce fermentation time. The use of single- and two-stage continuous cultures under micro-aerated conditions for simultaneous consumption of xylose and glucose, and fermentation to ethanol by ethanologenic Escherichia coli strain MSO4 was studied. Mineral medium supplemented with glucose, xylose and sodium acetate, was used to compare continuous cultures performance to batch cultures.
\end{abstract}

Results: Single-stage continuous cultures under micro-aerated conditions allowed the total co-consumption of a mixture of glucose and xylose ( 7.5 and $42.5 \mathrm{~g} / \mathrm{L}$, respectively) in mineral medium, with steady state ethanol production of $18 \mathrm{~g} / \mathrm{L}$, and a volumetric ethanol productivity of $0.9 \mathrm{~g} / \mathrm{L} h$, when low dilution rates $\left(0.05 \mathrm{~h}^{-1}\right)$ were used. However, the volumetric productivity was lower than the batch process under similar conditions (1.3 g/L h). Conversely, micro-aerated two-stage continuous culture enhanced the volumetric productivity up to $1.6 \mathrm{~g} / \mathrm{L} \mathrm{h}$ at a dilution rate of $0.15 \mathrm{~h}^{-1}$, with a total consumption of sugars and a slight reduction of the overall ethanol yield.

Conclusions: The total and simultaneous consumption of glucose and xylose by the ethanologenic E. coli strain MSO4 was accomplished by using two-stage continuous culture under micro-aerated conditions with an increase in the volumetric ethanol productivity of $23 \%$ and $78 \%$ when compared to batch and single-stage continuous cultures, respectively. Multi-stage continuous cultivation can be used to promote the simultaneous consumption of all sugars contained in biomass hydrolysates, and thus increase the volumetric ethanol productivity of the fermentation process.

Keywords: Ethanol, Continuous culture, Simultaneous co-fermentation, Escherichia coli, Xylose, Glucose, Serial bioreactors, Volumetric ethanol productivity

\footnotetext{
*Correspondence: alfredo@ibt.unam.mx

1 Departamento de Ingeniería Celular y Biocatálisis, Instituto de Biotecnología, Universidad Nacional Autónoma de México, Av.

Universidad 2001, Colonia Chamilpa, 62210 Cuernavaca, Morelos, Mexico

Full list of author information is available at the end of the article
} 


\section{Background}

Ethanol is the foremost biotechnological commodity produced worldwide, and the most widely used biofuel nowadays, [1-3]. Also, ethanol combustion is cleaner than gasoline and more efficient, since it increases octane levels, helping to reduce the air pollutant emissions and carbon dioxide net emissions, because of better oxidation of hydrocarbons [3-5]. Ethanol is currently produced from different food crops in the so-called first-generation bioethanol (FGB) [3, 6], but, due to several drawbacks with the use of such substrates, second-generation bioethanol (SGB) has emerged as an alternative to make use of the abundant, renewable, and inexpensive lignocellulosic biomass. Lignocellulosic biomass is composed of different polysaccharides, such as cellulose and hemicellulose, which can be a source of several fermentable sugars, of which xylose and glucose are the predominant sugars [4,7]; in addition to several inhibitors derived from the transformation of sugars and lignin, from which acetate is present in a higher concentration than the others $[8,9]$. After obtaining these syrups the efficient and fast utilization of pentose and hexose sugars, preferably simultaneously, is advantageous [10-14].

Traditionally, wild-type microorganisms have been used at industrial scale to produce FGB. However, these microorganisms are not able to efficiently ferment pentose sugars into SGB [10,15-17], which is a significant disadvantage because pentose sugars may constitute up to $40 \%$ of the total sugars in the biomass $[4,7,13,18]$. Due to these constraints, several microorganisms, such as yeasts and bacteria have been isolated or genetically modified, like Escherichia coli, Saccharomyces cerevisiae and Zymomonas mobilis, among others, to generate new biocatalysts capable to consume pentose and hexose sugar mixtures, for efficiently producing SGB [19-23]. For such purpose, E. coli shows several advantages, such as efficient consumption of hexoses and pentoses present in the hemicellulosic hydrolysates $[24,25]$ and high tolerance to various toxic compounds $[9,19,22,26]$. Despite these advantages, E. coli, and other wild-type and metabolic engineered ethanologenic microorganisms, show the phenomenon of carbon catabolite repression (CCR), which is a regulatory system used by different microorganisms for the utilization of a preferred carbon source $[7,25,27]$. In the case of ethanologenic E. coli growing in mineral media with a mixture of sugars or biomass hydrolysates, glucose is always consumed first, and then arabinose, xylose, and other sugars are utilized after glucose depletion $[7,28,29]$, causing a sequential utilization of mixed sugars, and often resulting in a delay and incomplete consumption of secondary sugars. This phenomenon limits ethanol titer, overall yield, and productivity $[7,13,25,28]$.
To overcome the phenomenon of CCR, strategies such as strain selection or metabolic engineering have been developed to engineer strains that can co-ferment mixtures of sugars simultaneously [7, 25, 28]. However, these strategies cause pleiotropic effects, such as significant reductions in the growth rate. An alternative to reduce or eliminate the effect of $\mathrm{CCR}$, when a mixture of sugars is used in the culture medium, is the use of cell culture strategies such as continuous culture. Continuous cultures, besides being used as a tool to select novel strains through adaptive evolution, are also used for other purposes. For instance, it is used to maintain process variables in a steady state and perform omics analysis: fluxomic, transcriptomic or proteomic. For production purposes it is also utilized to increase and extend the volumetric and specific productivities, and yields of relevant metabolites [30-32]. In this regard, single- and multi-stage continuous cultures are widely used in the commercial production of FGB [15, 18, 21, 32-34], however, little has been reported regarding the use of continuous culture to ferment mixtures of sugars (pentoses and hexoses). Furthermore, continuous culture, as well as fed-batch cultures, has potential advantages over batch cultures as the specific growth rate can be controlled, a higher and long-term volumetric productivity can be obtained through manipulation of the feeding rate, and low concentrations of substrates and metabolic product inhibitors, can be maintained in the bioreactor to reduce inhibition of growth. In addition, continuous cultures offer reduced downtime for cleaning, filling, and sterilization, which can be translated into the use of smaller fermenters volumes and plant size, lower capital investments, and the reduction of production costs, once the steady-state is reached, compared to batch and fed-batch cultures $[15,18,31,32]$. However, this culture method can also show some drawbacks such as: increased needs for process control and operation to optimize process conditions to reach the desired productivity; increased susceptibility to microbial contamination; the possibility of generating mutants or revertant strains (genetic instability) when the system is operated for a long time $[15,18,34]$; and the low concentration of ethanol resulting from a continuous process is particularly problematic given the cost for downstream processing, i.e. distillation.

This work aimed to evaluate the use of single- and twostage continuous cultures under micro-aerated conditions on the simultaneous consumption of glucose and xylose, ethanol production, and volumetric ethanol productivity by the ethanologenic strain $E$. coli MS04. The experiments were performed in mineral medium supplemented with xylose, glucose, and acetate, simulating the composition of acid diluted biomass hydrolysates generated from the hemicellulosic fraction of lignocellulosic 
biomass. The results showed that two-stage continuous culture was better than batch and single-stage continuous culture regarding the volumetric ethanol productivity by ethanologenic E. coli MS04, promoting the simultaneous and total consumption of pentose and hexose sugars to potentially produce SGB.

\section{Methods}

\section{Microorganism and media composition}

The ethanologenic strain used in this study was $E$. coli strain MS04 (E. coli MG1655: $\triangle p f l B, \triangle a d h E, \triangle f r d A$, $\triangle l d h A, \Delta x y l F G H$, gatC S184L, $\Delta$ reg 27.3kb, PpflB::pdc ${ }_{Z m^{-}}$ $a d h B_{Z m}$ ) which was engineered to produce ethanol as the primary product [22]. The mineral medium used in this work was the modified AM1 mineral medium [22], which was used in batch, single- and two-stage continuous experiments carried out in this study.

\section{Inoculum preparation}

Seed cultures of strain MS04 were prepared by inoculating $1.5 \mathrm{~mL}$ of a frozen stock $(40 \mathrm{w} / \mathrm{w}$ glycerol solution and cells grown in AM1 medium) into $100 \mathrm{~mL}$ of AM1 medium supplemented with glucose $(7.5 \mathrm{~g} / \mathrm{L})$, xylose $(42.5 \mathrm{~g} / \mathrm{L})$ and sodium acetate $(2 \mathrm{~g} / \mathrm{L})$ in a $500-\mathrm{mL}$ baffled shake flask, which was incubated at $37{ }^{\circ} \mathrm{C}$ with an initial $\mathrm{pH}$ of 7.0 and $300 \mathrm{rpm}$. After $12 \mathrm{~h}$ of cultivation, cells were harvested by centrifugation $(10,000 \times g, 10 \mathrm{~min}$, $4{ }^{\circ} \mathrm{C}$ ), and resuspended into the same mineral medium to start a batch culture, which had an initial inoculum equivalent to 0.1 optical density at $600 \mathrm{~nm}\left(\mathrm{OD}_{600}\right)$.

\section{Batch culture under micro-aerated conditions}

Batch cultures were performed using a working volume of $750 \mathrm{~mL}, \mathrm{pH}$ controlled at $7.0,37{ }^{\circ} \mathrm{C}, 400 \mathrm{rpm}$, $0.1 \mathrm{vvm}$ of air, corresponding to a volumetric oxygen transfer coefficient $\left(k_{\mathrm{L}} \mathrm{a}\right)$ of $7.2 \mathrm{~h}^{-1}$ [35]. Oxygen tension was measured using a sterilizable dissolved oxygen electrode (Ingold, model A420), as previously described [35]. Mineral medium AM1 [36] supplemented with glucose $(7.5 \mathrm{~g} / \mathrm{L})$, xylose $(42.5 \mathrm{~g} / \mathrm{L})$, and sodium acetate $(2 \mathrm{~g} / \mathrm{L})$ was used in this study to simulate the composition of diluted hemicellulosic hydrolysates [37, 38]. The concentrations of sugars and acetate previously mentioned were also chosen to maintain a concentration of solutes where cells were not drastically inhibited by osmotic effects, since it is already known that bacteria can be inhibited by carbon sources at concentrations higher than $50 \mathrm{~g} / \mathrm{L}$. The mineral medium was prepared with sugars, and salts solutions sterilized separately by filtration and added to the bioreactor aseptically before inoculation. After $2 \mathrm{~h}$ of inoculation, the oxygen tension dropped to $0 \%$ of the air saturation.
Single-stage continuous culture experiments under micro-aerated conditions

Single-stage continuous cultures (SSCC) were conducted by keeping the same medium composition and process variables as those used in batch culture experiments. SSCC were started as a batch culture, inoculated to an $\mathrm{OD}_{600}$ of 0.1 , and were allowed to run for $12 \mathrm{~h}$. Afterwards, the bioreactor was set up on continuous culture mode, by switching on a pre-calibrated peristaltic feed pump to supply sterile feed medium from the feed medium reservoir. To maintain a constant liquid culture volume $(\mathrm{V}=750 \mathrm{~mL})$, spent medium was continuously withdrawn at the same flow rate than the feed pump. Various feed flow rates $(\mathrm{F} ; \mathrm{L} / \mathrm{h})$ were tested, thus evaluating different dilution rates $(\mathrm{D}=\mathrm{F} / \mathrm{V})$ [31], which are equal to the specific growth rate $\left(D=\mu ; h^{-1}\right)$ at steady state.

Five fixed D were evaluated in this culture mode: 0.05 , $0.1,0.15,0.2$ and $0.3 \mathrm{~h}^{-1}$. Steady-state was assumed when the cell, sugars, and ethanol concentrations did not change over time after three successive samplings, which was generally when the system ran for a period corresponding to 3-5 times the liquid residence time $\left(\theta_{c}=1 / D\right)$ [31]. Culture samples were analyzed immediately to determine cell, residual sugars, and ethanol concentrations.

\section{Two-stage continuous culture experiments under micro-aerated conditions}

To evaluate the consumption of glucose and xylose, and the production and productivity of ethanol in multistage continuous cultivations, two-stage continuous cultures (TSCC) were performed. Two fermenters were connected in series with a working volume of $750 \mathrm{~mL}$ each (combined working volume of $1.5 \mathrm{~L}$ ) and were fed with fresh mineral medium only in the first stage, thus evaluating the same $D$ in both stages $\left(D_{1}=D_{2}\right)$. Medium composition and process variables were the same as single-stage continuous culture. The first stage of the TSCC was initiated as previously described with the singlestage continuous culture, but the effluent stream from this stage was sent to the subsequent second stage with a peristaltic pump, and then the spent medium from the second stage was transferred to the waste bottle using a third outlet pump. Four fixed dilution rates were evaluated in both stages $\left(0.1,0.15,0.2\right.$ and $\left.0.3 \mathrm{~h}^{-1}\right)$. Again, the steady-state was assumed when cell, sugars and ethanol concentrations did not change over time in both stages $\left(3-5 \theta_{c}\right)$. Unlike SSCC, the specific growth rate at the second stage $\left(\mu_{2}\right)$ was always lower than the dilution rate at the same stage $\left(D_{2}\right)$ because of the entry of biomass in the second stage, coming from the first stage. The $\mu_{2}$ was 
calculated according to Eq. 1, which is derived from the mass balances on TSCC.

$$
\mu_{2}=\frac{D_{2}\left(x_{2}-x_{1}\right)}{x_{2}}
$$

where $x_{1}$ and $x_{2}$ are the steady-state cell concentrations at the first and second stage of TSCC, respectively.

\section{Analytical methods}

Cell concentration was determined spectrophotometrically as optical density at $600 \mathrm{~nm}$ (DU-70, Beckman Instruments, Inc. Fullerton, CA), and converted to dry cell weight $(\mathrm{DCW})$ per liter using a calibration curve (1 optical density at $\left.600 \mathrm{~nm}=0.37 \mathrm{~g}_{\mathrm{DCW}} / \mathrm{L}\right)$. Glucose and xylose concentrations were measured with a biochemical analyzer (YSI model 2700, YSI Inc., Yellow Springs, $\mathrm{OH}$ ); whereas acetate concentrations were measured by high-performance liquid chromatography [22]. Ethanol concentrations were measured by gas chromatography using $n$-butanol (1\%) as an internal standard (6850 Series GC System, Agilent, Wilmington, DE, USA) as previously reported [22]. The volumetric ethanol productivity $\left(\mathrm{Q}_{\mathrm{P}}\right)$ in the SSCC and TSCC was calculated as the product of the operating dilution rate and ethanol concentration produced at the first (Eq. 2) and the second stage (Eq. 3), respectively. The specific ethanol productivity $\left(\mathrm{q}_{\mathrm{p}}\right)$ in each stage was calculated as the product of the specific growth rate $(\mu)$ and the specific product yield $\left(\mathrm{Y}_{\mathrm{PX}}\right)$ at the steady state (Eq. 4). Theoretical ethanol yields on sugars consumed $\left(\mathrm{Y}_{\mathrm{PS}}\right)$ in each stage in continuous culture, as well as in batch culture, were calculated as previously described [35], and are expressed as a percentage of the theoretical maximum yield $\left(0.51 \mathrm{~g}_{\text {ethanol }} / \mathrm{g}_{\text {sugar }}\right)$.

$$
\begin{aligned}
& Q_{P 1}=D_{1} P_{1} \\
& Q_{P 2}=D_{2}\left(P_{2}-P_{1}\right) \\
& q_{P}=\mu Y_{P X}
\end{aligned}
$$

\section{Results and discussion}

Batch culture with the ethanologenic strain E. coli MSO4 under micro-aerated conditions displays sequential consumption of glucose and xylose

Ethanologenic E. coli strain MS04 was previously engineered and evolved to produce ethanol as the primary fermentation product from hexose and pentose sugars in the presence of high concentrations of acetate [22]. As previously reported [22], compared to the absence of acetate, concentrations of this acid ranging from 2 to $10 \mathrm{~g} / \mathrm{L}$ promote an increase in the specific growth rate, cell mass formation, and ethanol volumetric productivity. This is probably due to an increase in the synthesis of acetylCoA from acetate because the genes for its catabolism

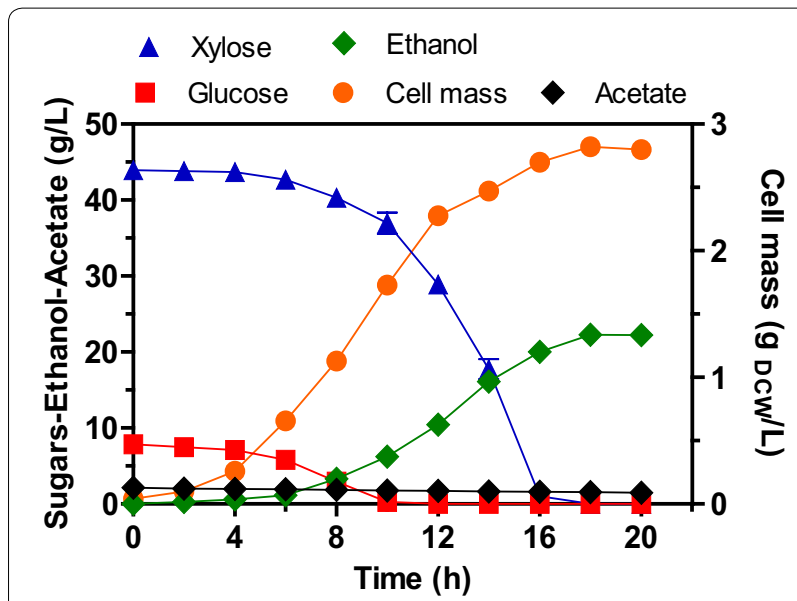

Fig. 1 Growth, sugar consumption and acetate, and ethanol production kinetics by the engineered strain E. coli MSO4 in micro-aerated batch culture using mineral medium supplemented with xylose, glucose and sodium acetate $(42.5,7.5$ and $2 \mathrm{~g} / \mathrm{L}$, respectively)

were not interrupted and the adaptive evolution process improved acetate tolerance in strain MS04 [22]. In batch culture, the total concentration of sugars $(50 \mathrm{~g} / \mathrm{L})$ was consumed in $18 \mathrm{~h}$, with an ethanol production of $22 \mathrm{~g} / \mathrm{L}$, and a cell mass concentration close to $3 \mathrm{~g}_{\mathrm{DCW}} / \mathrm{L}$ (Fig. 1). The consumption of sugars at the beginning of the culture was semi-sequential, with glucose being consumed during the first $6 \mathrm{~h}$, followed by a period of simultaneous consumption of glucose and xylose (from 6 to $10 \mathrm{~h}$ of culture), until depletion of the former and then xylose being depleted between 16 and $18 \mathrm{~h}$. Kinetic parameters, such as $\mu$ and $\mathrm{q}_{\mathrm{P}}$ at the exponential growth phase, as well as $Y_{\mathrm{PS}}, \mathrm{Y}_{\mathrm{XS}}, \mathrm{Q}_{\mathrm{P}}$ and carbon balance were estimated at the global phase and are shown in Table 1. A significant fraction of the carbon was directed towards the production of ethanol, with a minimal amount directed to synthesize cells or other by-products (Table 1). For instance, the cell yield on consumed sugars $\left(\mathrm{Y}_{\mathrm{XS}}\right)$ was only $0.054 \mathrm{~g} / \mathrm{g}$, there was a negligible production of acetate (Fig. 1), and a carbon recovery of $91 \%$ was obtained. About $25 \%$ of the acetate present initially in the medium was consumed, being this quantity probably used for biosynthetic pathways [22], whereas the residual concentration $(1.5 \mathrm{~g} / \mathrm{L})$ remained constant throughout the culture.

Ethanologenic E. coli MS04 showed a high $\mu$ $\left(0.46 \mathrm{~h}^{-1}\right)$ when it grew in the presence of a xylose/glucose mixture $(50 \mathrm{~g} / \mathrm{L})$ in mineral medium added with sodium acetate, as well as a $Y_{P S}$ close to $90 \%$ of the theoretical maximum, and a high $\mathrm{Q}_{\mathrm{P}}$ and $\mathrm{q}_{\mathrm{P}}(1.3 \mathrm{~g} / \mathrm{L} \mathrm{h}$ and $1.38 \mathrm{~g} / \mathrm{g} \mathrm{h}$, respectively; Table 1 ). The kinetic and stoichiometric parameters of the strain MS04 are among the highest reported by ethanologenic microorganisms, 
Table 1 Kinetic and stoichiometric parameters of ethanologenic E. coli MSO4 in batch and SSCC under micro-aerated conditions in mineral medium supplemented with xylose $(42.5 \mathrm{~g} / \mathrm{L})$, glucose $(7.5 \mathrm{~g} / \mathrm{L})$, and sodium acetate $(2 \mathrm{~g} / \mathrm{L})$

\begin{tabular}{lllllllll}
\hline Culture & $\mathbf{D}\left(\mathbf{h}^{-\mathbf{1}}\right)$ & $\boldsymbol{\mu}\left(\mathbf{h}^{-\mathbf{1}}\right)$ & Ethanol $\left(\mathbf{g}_{\mathrm{EtOH}} / \mathbf{L}\right)$ & $\mathbf{Y}_{\mathbf{P S}}(\%, \mathbf{w} / \mathbf{w})$ & $\mathbf{Q}_{\mathbf{p}}\left(\mathbf{g}_{\mathrm{EtOH}} / \mathbf{L} \mathbf{h}\right)$ & $\mathbf{q}_{\mathbf{p}}\left(\mathbf{g}_{\mathrm{EtOH}} / \mathbf{g}_{\mathbf{D C W}} \mathbf{h}\right)$ & $\mathbf{Y}_{\mathbf{x s}}\left(\mathbf{g}_{\mathbf{D C w}} / \mathbf{g}_{\mathbf{S}}\right)$ & $\mathbf{C a r b o n} \mathbf{b a l a n c e}(\%)$ \\
\hline Batch & - & $0.46 \pm 0.01$ & $22.2 \pm 0.09$ & $87 \pm 1.2$ & $1.3 \pm 0.05$ & $1.38 \pm 0.02$ & $0.054 \pm 0.001$ & $91 \pm 0.91$ \\
SSCC & 0.05 & 0.05 & $18.2 \pm 0.15$ & $72 \pm 0.6$ & $0.9 \pm 0.01$ & $0.58 \pm 0.03$ & $0.032 \pm 0.002$ & $75 \pm 0.55$ \\
SSCC & 0.10 & 0.10 & $15 \pm 0.4$ & $82 \pm 1$ & $1.5 \pm 0.04$ & $0.81 \pm 0.04$ & $0.051 \pm 0.002$ & $91 \pm 0.55$ \\
SSCC & 0.15 & 0.15 & $10 \pm 0.2$ & $82 \pm 2$ & $1.5 \pm 0.04$ & $0.99 \pm 0.02$ & $0.064 \pm 0.005$ & $95 \pm 1.3$ \\
SSCC & 0.20 & 0.20 & $5.1 \pm 0.2$ & $77 \pm 2$ & $1.0 \pm 0.04$ & $1.04 \pm 0.03$ & $0.076 \pm 0.003$ & $96 \pm 0.7$ \\
SSCC & 0.30 & 0.30 & $1.8 \pm 0.2$ & $80 \pm 2$ & $0.53 \pm 0.06$ & $1.47 \pm 0.02$ & $0.087 \pm 0.014$ & $99 \pm 0.5$ \\
\hline
\end{tabular}

Average values and standard errors are shown from duplicate experiments for batch cultures and triplicate measurements during the steady state of continuous cultures

$D$ dilution rate, $\mu$ specific growth rate, $Y_{P S}$ ethanol yield on sugars consumed as percentage of the maximum theoretical; $Y_{X S}$ biomass yield on sugars consumed, $Q_{P}$ volumetric ethanol productivity, $q_{p}$ specific ethanol productivity

such as strains of E. coli and S. cerevisiae, growing in batch culture on synthetic media supplemented with xylose or glucose/xylose mixtures [39-43]. Even though there are differences among the studies, such as the metabolic background of each strain, the culture media, the implementation of metabolic evolution processes, the process conditions (aerated or non-aerated) used in each case, among others [39-43]. In the case of strain MS04, the rate of xylose transport into the cell, and/or the metabolic flux to produce ethanol may be higher, and therefore the strain MS04 can metabolize xylose and produce ethanol at higher rates in comparison to other ethanologenic bacteria or yeast strains [39-43].

On the other hand, process conditions, such as agitation and aeration could also influence the productivity of the process. The main variable in this study was the oxygen transfer rate, which depends on the agitation and aeration. The aerated conditions used in this study $\left(0.1 \mathrm{vvm}, 400 \mathrm{rpm}, k_{\mathrm{L}} \mathrm{a}=7.2 \mathrm{~h}^{-1}\right)$ were previously described as optimal for the consumption of glucose/xylose mixtures, and production of ethanol by $E$. coli MS04 [35]. Therefore, high values of $\mu, \mathrm{q}_{\mathrm{P}}$, and $\mathrm{Q}_{\mathrm{P}}$ $(1.3 \mathrm{~g} / \mathrm{L} \mathrm{h})$ were reached in this study compared to the parameters found using other ethanologenic strains of $E$. coli and $S$. cerevisiae metabolizing xylose/glucose mixtures $\left(\mathrm{Q}_{\mathrm{P}}\right.$ from 0.55 to $\left.0.92 \mathrm{~g} / \mathrm{L} \mathrm{h}\right)$, where no air was supplied to the media, or even anaerobic conditions were maintained in the culture [39, 43-45].

Even though strain MS04 shows relevant advantages with respect to the production of ethanol from glucose/ xylose mixtures in batch culture, the phenomenon of catabolite repression by glucose is still present, avoiding the co-fermentation of xylose until the concentration of glucose in the medium was lower than $6 \mathrm{~g} / \mathrm{L}$ (Fig. 1), thus diminishing the volumetric ethanol productivity and the overall ethanol yield. Therefore, as proposed above, the use of continuous culture could

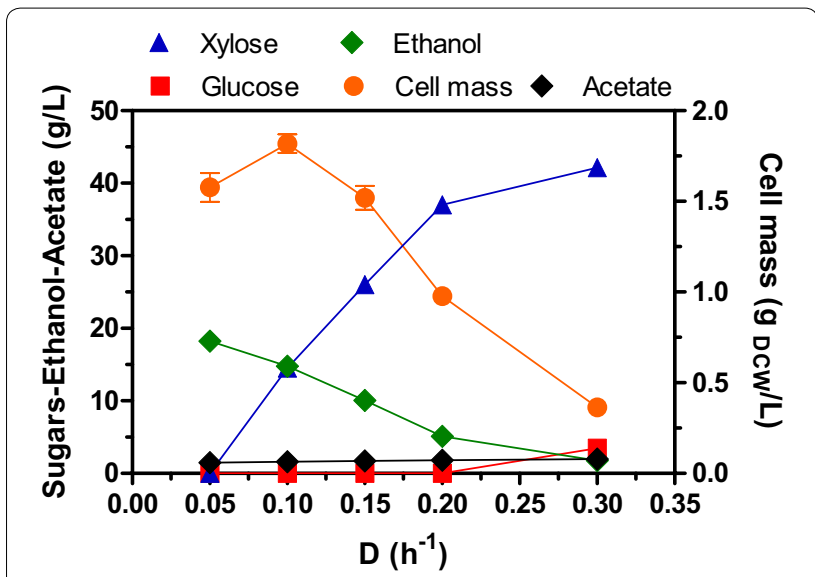

Fig. 2 Cell mass, residual sugars and acetate, and ethanol concentration at steady-state of SSCC under micro-aerated conditions at different values of D by E. coli MS04. Feeding medium as described in Fig. 1

provide a release from the catabolite repression, and thus increase the rate of consumption of sugars and ethanol productivity.

\section{Single-stage continuous culture under micro-aerated conditions promotes the total and simultaneous consumption of glucose and xylose at low specific growth rates}

Continuous culture experiments were carried out to determine if this condition would promote the simultaneous consumption of sugars (glucose and xylose) and increase the volumetric productivity with a minimum effect on the ethanol yield compared to batch culture.

The results of cell mass, residual sugars, and ethanol concentrations by the strain MS04 at the steady-state of SSCC are shown in Fig. 2, where a complete consumption of the glucose/xylose mixture $(7.5 / 42.5 \mathrm{~g} / \mathrm{L}$ respectively) and the highest ethanol concentration $(18.2 \mathrm{~g} / \mathrm{L})$ 
was obtained at the lowest $\mathrm{D}\left(0.05 \mathrm{~h}^{-1}\right)$ tested (Table 1$)$. Similarly to results found in the batch experiments, about $0.5 \mathrm{~g} / \mathrm{L}$ of acetate was consumed at steady state of all D's tested in SSCC (Fig. 2). As D increased, the simultaneous consumption of the glucose/xylose mixture was also observed, with the total consumption of glucose $(7.5 \mathrm{~g} / \mathrm{L})$, and the consumption of xylose ranging from 5.5 to $28 \mathrm{~g} / \mathrm{L}$ at D's $=0.1-0.2 \mathrm{~h}^{-1}$. At D of $0.3 \mathrm{~h}^{-1}$, only a small concentration of glucose and xylose was consumed ( 4 and $0.4 \mathrm{~g} / \mathrm{L}$, respectively), and cell mass and ethanol production were reduced to $0.36 \mathrm{~g}_{\mathrm{DCW}} / \mathrm{L}$ and $1.8 \mathrm{~g} / \mathrm{L}$, respectively (Fig. 2). At this D, approximately $3.5 \mathrm{~g} / \mathrm{L}$ of glucose remained in the culture broth, slowing the xylose consumption substantially due to the catabolic repression effect exerted by glucose. Furthermore, the specific growth rate of strain MS04 cultured in similar conditions but using batch cultivations with xylose $(50 \mathrm{~g} / \mathrm{L})$ as the sole carbon source was in the range of 0.21 to $0.25 \mathrm{~h}^{-1}$ (data not shown), i.e. 54-45\% lower than that found with the glucose-xylose mixture in batch fermentations. However, at all dilutions tested, there was co-fermentation of glucose and xylose at different ratios in a single stage, which was influenced only by the dilution rate used in the experiments. In all cases, the concentration of cell mass was lower than that obtained in the batch cultures $\left(<2 \mathrm{~g}_{\mathrm{DCW}} / \mathrm{L}\right)$, probably due to the dilution effect and the inhibitory effect of ethanol produced. Concerning the $Q_{p}$, the highest value $(1.5 \mathrm{~g} / \mathrm{L} \mathrm{h})$ was reached at $\mathrm{D}=0.1$ and $0.15 \mathrm{~h}^{-1}$, which was higher than that obtained in batch culture (Table 1), though xylose was not totally consumed at these conditions (Fig. 2). The $\mathrm{q}_{\mathrm{p}}$ had a direct relationship with $\mathrm{D}$, and increased from 0.58 to $1.47 \mathrm{~g} / \mathrm{g} \mathrm{h}$, in the range of $\mathrm{D}=0.05-0.3 \mathrm{~h}^{-1}$ (Table 1). Furthermore, the $\mathrm{Y}_{\mathrm{PS}}$ in SSCC was maintained practically constant $(\approx 80 \%)$ in the range of $D=0.1-0.3 \mathrm{~h}^{-1}$, and only diminished to $70 \%$ when the $\mathrm{D}$ was $0.05 \mathrm{~h}^{-1}$ (Table 1 ). With respect to the $\mathrm{Y}_{\mathrm{XS}}$ and carbon balance, it was observed that both parameters had also a direct relationship with $\mathrm{D}$, showing that at low dilutions less carbon was directed to the synthesis of biomass, probably due to the redirection of substrate consumption for cellular maintenance processes, under the stress conditions derived from the high concentrations of ethanol (Table 1).

Comparing the results obtained in SSCC with respect to batch culture under the same process conditions, SSCC was superior in terms of volumetric and specific ethanol productivities, which increased approximately $20 \%$ and $7 \%$, at $\mathrm{D}=0.1-0.15 \mathrm{~h}^{-1}$, and $\mathrm{D}=0.3 \mathrm{~h}^{-1}$, respectively (Table 1 ). The opposite was observed for the case of titer and yield, where these parameters were lower, at all conditions tested, to those obtained in batch culture (Table 1). This kind of behavior is commonly observed in SSCC, where higher $\mathrm{Q}_{\mathrm{P}}$ and $\mathrm{q}_{\mathrm{P}}$ are reached at an optimum D; while titer, yield and, the carbon balance are lower at low dilutions in comparison to batch culture $[15,18,44,46]$. The reduction in the overall ethanol yield and carbon balance at $\mathrm{D}=0.05 \mathrm{~h}^{-1}$, but with the higher concentration of ethanol achieved (18 g/L) (Table 1), suggest that some carbon was lost as $\mathrm{CO}_{2}$. Finally, because of the micro-aerated conditions used in this study $\left(k_{\mathrm{L}} \mathrm{a}=7.2 \mathrm{~h}^{-1}\right)$, it is also possible that a certain amount of $\mathrm{CO}_{2}$ was released from the microaerobic metabolism in the tricarboxylic acid cycle, which was not quantified at the exit of the fermentor. In this respect, this behavior has also been observed with other ethanologenic $E$. coli strains, such as ATCC 11303 and FBR5 (both transformed with the plasmid pLOI297), which were grown in batch and SSCC, and both produced lower ethanol titer and yield in SSCC compared to the values reached in batch culture under the same conditions $[18,44]$. Lower ethanol yields in SSCC were also obtained [46] (about $90 \%$ of the theoretical) when two immobilized ethanologenic E. coli strains (AFF01 and CT1101) were cocultured to convert glucose/xylose mixtures to ethanol, compared to batch culture, where a yield of more than 95\% was achieved [46]. The authors proposed that the lower yield in SSCC was due to the continuous exposure of immobilized cells to high concentrations of ethanol, unlike when the cells were grown in batch culture, where the exposition to high ethanol concentrations was only present at the end of the culture when the sugars were exhausted. They also suggested that the incomplete utilization of sugars in continuous culture by the immobilized cells was because of the inhibition of ethanol [46]. Thus, even with the possible inhibition of ethanol on growth and ethanol production by MS04, the performance of strain MS04 simultaneously fermenting mixed sugars to ethanol in SSCC with mineral medium is among the best reported so far, considering the sugar conversion, and the specific and volumetric productivities reached using mineral or complex media by other ethanologenic bacteria and yeast strains $[18,33,44-50]$.

\section{Two-stage continuous culture promotes the total and simultaneous consumption of sugars allowing to achieve a high volumetric ethanol productivity}

To determine if it would be possible to maintain high volumetric ethanol productivities in continuous culture with a higher consumption of sugars and production of ethanol in the system, two-stage continuous cultures (TSCC), under micro-aerated conditions $\left(k_{\mathrm{L}} \mathrm{a}=7.2 \mathrm{~h}^{-1}\right)$, were performed to consume the residual sugars exiting the SSCC when D was $>0.05 \mathrm{~h}^{-1}$. The working volume and $\mathrm{D}$ were the same in both stages of the TSCC. Four values of D were tested (0.1, 0.15, 0.2 and $0.3 \mathrm{~h}^{-1}$ ), feeding AM1 mineral medium 


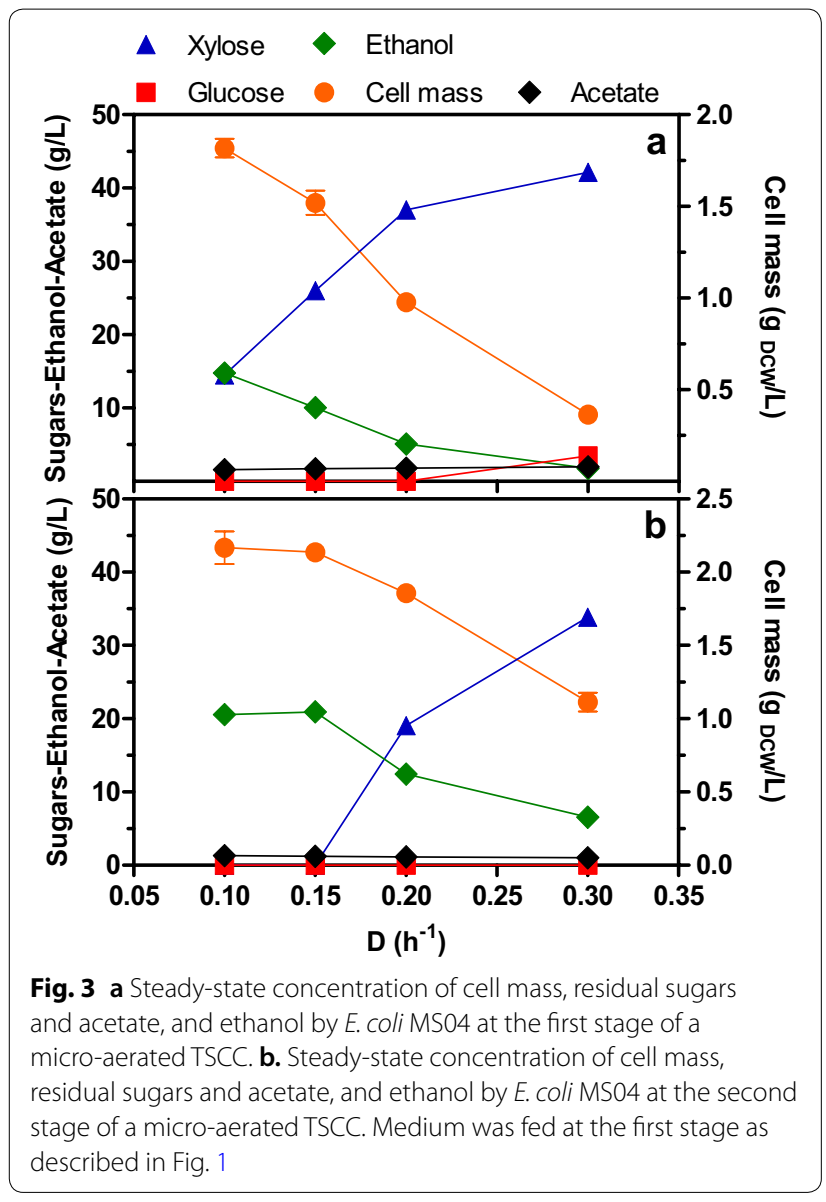

supplemented with xylose, glucose and sodium acetate $(42.5,7.5$ and $2 \mathrm{~g} / \mathrm{L}$ respectively) only to the first stage. Figure $3 \mathrm{a}, \mathrm{b}$ show the concentrations, at the steady state, of cell mass, residual sugars (xylose and glucose), acetate and ethanol at the first and second stage, respectively, in the TSCC at different values of D. As mentioned before, the first stage of TSCC had the same behavior as SSCC, with the presence of residual sugars in the effluent at $D_{1} \geq 0.1 \mathrm{~h}^{-1}$, and an inverse relationship between $D_{1}$ and cell mass, ethanol production, consumed sugars and acetate (Fig. 3a). The highest value of $\mathrm{Q}_{\mathrm{P}}(\sim 1.5 \mathrm{~g} / \mathrm{L} \mathrm{h})$ was attained at $\mathrm{D}_{1}$ of $0.1 \mathrm{~h}^{-1}$ and $0.15 \mathrm{~h}^{-1}$ and decreased as $\mathrm{D}_{1}$ increased (Table 2). The highest $\mathrm{Y}_{\mathrm{PS}}$ was $82 \%$ at a $\mathrm{D}_{1}$ of $0.15 \mathrm{~h}^{-1}$ and decreased to about $80 \%$ at the other $\mathrm{D}_{1}{ }^{\prime} \mathrm{s}$ tested (Table 2).

For the second stage of TSCC, operated at the same D as the first stage, at a $D_{2}$ of 0.1 and $0.15 \mathrm{~h}^{-1}$, the remaining xylose concentration leaving the first stage (15 and $29 \mathrm{~g} / \mathrm{L}$, respectively) was completely consumed, and only when the system was operated at $\mathrm{D}_{2} \geq 0.2 \mathrm{~h}^{-1}$, xylose was present in the effluent medium up to a concentration of $34 \mathrm{~g} / \mathrm{L}$ (Fig. 3b). The maximum concentration of ethanol reached at the second stage was $21 \mathrm{~g} / \mathrm{L}$ at $\mathrm{D}_{2}=0.1$ and $0.15 \mathrm{~h}^{-1}$, and decreased to $7 \mathrm{~g} / \mathrm{L}$ as $\mathrm{D}_{2}$ increased to $0.3 \mathrm{~h}^{-1}$ (Fig. 3b). Maximum cell mass concentration of $2.2 \mathrm{~g}_{\mathrm{DCW}} / \mathrm{L}$ was attained at the lowest $\mathrm{D}_{2}\left(0.1 \mathrm{~h}^{-1}\right)$, which was lower than the concentration reached in batch culture $\left(2.8 \mathrm{~g}_{\mathrm{DCW}} / \mathrm{L}\right)$, and it also had an inverse relationship with $\mathrm{D}_{2}$, as in the case of the first stage (Fig. 3b).

On the other hand, the concentration of acetate consumed in the second stage was similar to that consumed in the first stage $(\sim 0.5 \mathrm{~g} / \mathrm{L})$. At the exit of the second stage, there was a residual concentration of acetate $\geq 1 \mathrm{~g} / \mathrm{L}$, therefore, the acetate present in the feed medium was not completely consumed in the second stage. As previously reported [22], we suggest that low amounts of acetate are necessary for the synthesis of acetyl-CoA and other biosynthetic molecules. $\mathrm{Q}_{\mathrm{p}}$ reached a maximum of $1.6 \mathrm{~g} / \mathrm{L} \mathrm{h}$ at $\mathrm{D}_{2}=0.15 \mathrm{~h}^{-1}$, followed by the conditions when $\mathrm{D}_{2} \geq 0.2 \mathrm{~h}^{-1}$, with the lowest value $(0.6 \mathrm{~g} / \mathrm{L} \mathrm{h})$ obtained at $D_{2}=0.1 \mathrm{~h}^{-1}$ (Table 2). In turn, values of $Y_{P S}$ in the second stage were in the range of $78-82 \%$ of the theoretical for $\mathrm{D}_{2} \geq 0.1 \mathrm{~h}^{-1}$ (Table 2).

Table 2 Kinetic and stoichiometric parameters of the strain E. coli MS04 calculated at both stages in TSCC, and as a global TSCC (G) under micro-aerated conditions in mineral medium supplemented with xylose (42.5 g/L), g/ucose (7.5 g/L) and sodium acetate $(2 \mathrm{~g} / \mathrm{L})$

\begin{tabular}{|c|c|c|c|c|c|c|c|c|}
\hline$\mu_{1}\left(h^{-1}\right)$ & $\mu_{2}\left(h^{-1}\right)$ & $\mu_{G}\left(h^{-1}\right)$ & $\mathrm{Q}_{\mathrm{P} 1}\left(\mathrm{~g}_{\mathrm{EtOH}} / \mathrm{L} \mathrm{h}\right)$ & $\mathrm{Y}_{\mathrm{PS} 1}(\%)$ & $\mathrm{Q}_{\mathrm{P2}}\left(\mathrm{g}_{\mathrm{EtOH}} / \mathrm{Lh}\right)$ & $\mathrm{Y}_{\mathrm{PS2}}(\%)$ & $\mathrm{Q}_{\mathrm{PG}}\left(\mathrm{g}_{\mathrm{EtOH}} / \mathrm{L} \mathrm{h}\right)$ & $\mathrm{Y}_{\text {PSG }}(\%)$ \\
\hline 0.10 & 0.018 & 0.050 & $1.48 \pm 0.04$ & $81 \pm 1.0$ & $0.58 \pm 0.04$ & $78 \pm 2.5$ & $1.0 \pm 0.01$ & $81 \pm 0.5$ \\
\hline 0.15 & 0.043 & 0.075 & $1.51 \pm 0.04$ & $82 \pm 0.8$ & $1.63 \pm 0.07$ & $82 \pm 1.5$ & $1.6 \pm 0.03$ & $82 \pm 0.9$ \\
\hline 0.20 & 0.095 & 0.100 & $1.00 \pm 0.04$ & $77 \pm 3.0$ & $1.46 \pm 0.09$ & $81 \pm 3.0$ & $1.2 \pm 0.05$ & $79 \pm 3.0$ \\
\hline 0.30 & 0.200 & 0.150 & $0.53 \pm 0.06$ & $80 \pm 3.5$ & $1.44 \pm 0.07$ & $80 \pm 3.5$ & $1.0 \pm 0.05$ & $80 \pm 3.0$ \\
\hline
\end{tabular}

Average values and standard errors are shown from triplicates

$\mu_{1}, \mu_{2}, \mu_{G}$ : specific growth rates at stage 1 and 2 of the two-stage continuous culture, and at the continuous global culture, respectively $\mathrm{Q}_{\mathrm{P} 1}, \mathrm{Q}_{\mathrm{P} 2}, \mathrm{Q}_{\mathrm{PG}}$ : ethanol volumetric productivity at stage 1 and 2 of the two-stage continuous culture, and at the continuous global culture, respectively $\mathrm{Y}_{\mathrm{PS} 1}, \mathrm{Y}_{\mathrm{PS} 2}, \mathrm{Y}_{\mathrm{PSG}}$ : ethanol overall yield at stage 1 and 2 of the two-stage continuous culture, and at the continuous global culture, respectively G: continuous global culture was considered as a single-stage continuous culture with the sum of volumes of both stages in the two-stage continuous culture and fed at the same flow rate. $D_{G}=D_{1} / 2=D_{2} / 2$ 
With the aim of evaluating the TSCC as a system, and not only as two fermenters connected in series, the process parameters of TSCC were estimated as a continuous global culture, where both stages are fused into a single stage continuous culture with the sum of working volumes of both stages $(1.5 \mathrm{~L})$ and fed at the same flow rate as each D was operated in TSCC. Under these conditions, the dilution rate of the continuous global culture $\left(D_{G}\right)$ was half of $D_{1}$ or $D_{2}$ in TSCC, and at the same time, $D_{G}$ was equal to the specific growth rate in the global continuous culture $\left(\mu_{\mathrm{G}}\right)$ (Table 2). The residual xylose and ethanol concentration in the global steady-state were the same as those in the steady-state of second-stage of TSCC, with the highest residual xylose concentration $(34 \mathrm{~g} / \mathrm{L})$ at $\mathrm{D}_{\mathrm{G}}=0.15 \mathrm{~h}^{-1}\left(\mathrm{D}_{1}=\mathrm{D}_{2}=0.3 \mathrm{~h}^{-1}\right)$, and a maximum concentration of ethanol of $21 \mathrm{~g} / \mathrm{L}$ at $\mathrm{D}_{\mathrm{G}}=0.05$ and $0.075 \mathrm{~h}^{-1}$. With these data, process parameters were calculated and are presented in Table 2, with a maximum $\mathrm{Y}_{\mathrm{PS}}$ of $82 \%$ at a $\mathrm{D}_{\mathrm{G}}$ of $0.075 \mathrm{~h}^{-1}$, and about $80 \%$ of the theoretical in the other $\mathrm{D}_{\mathrm{G}}{ }^{\prime} \mathrm{s}$. The maximum $\mathrm{Q}_{\mathrm{P}}$ achieved $\left(1.6 \mathrm{~g}_{\mathrm{EtOH}} / \mathrm{L} \mathrm{h}\right)$ was also obtained at a $\mathrm{D}_{\mathrm{G}}$ of $0.075 \mathrm{~h}^{-1}$, with an increase of $23 \%$ and $78 \%$ compared to batch culture and SSCC, respectively, and it diminished to 1.0 $\mathrm{g}_{\mathrm{EtOH}} / \mathrm{L} \mathrm{h}$ at a D of $0.3 \mathrm{~h}^{-1}$ (Table 2).

The advantages of using multi-stage continuous culture (MSCC) over batch and SSCC with respect to volumetric productivity, product concentration, and substrate utilization, have been documented elsewhere [15, 32, 34]. One of the main advantages of using MSCC instead of batch culture or SSCC is the feasibility of operating each stage separately and independently, with different process conditions, to find the optimal conditions in each stage to reach the highest substrate conversion, product concentration and productivity in the system [32]. Thus, when comparing a SSCC with an equivalent MSCC, both operated at the same flow rate, total working volume, and global D, the later will show better results regarding product concentration and productivity, thus improving the technical and economic feasibility of the process $[15,34]$.

In this study, we used the same D and working volume in each stage of the TSCC to ferment a mixture of sugars $(50 \mathrm{~g} / \mathrm{L})$, consisting of glucose and xylose, achieving the total conversion of sugars, and reaching a $Q_{p}$ of $1.6 \mathrm{~g} / \mathrm{L} \mathrm{h}$ at a $\mathrm{D}_{\mathrm{G}}$ of $0.075 \mathrm{~h}^{-1}$. These results are promising when considering the use of the mineral medium, the absence of antibiotics in the medium, and the use of a high xylose/ glucose ratio, in comparison to other results where complex media, the presence of antibiotics, and/or low xylose concentrations are used. Some authors have reported the use of TSCC to ferment mixed sugars to ethanol with ethanologenic yeast, bacteria, or by using both in a coculture, inoculating each in different stages. For instance, two recombinant $S$. cerevisiae strains, LNH33 and
LNH-ST, were separately cultivated in TSCC, with complex medium supplemented with xylose $(34 \mathrm{~g} / \mathrm{L})$ and glucose $(24 \mathrm{~g} / \mathrm{L})$, and a working volume of $1 \mathrm{~L}$ each, at D of $0.042-0.043 \mathrm{~h}^{-1}$ in both stages, under non-aerated conditions $[49,50]$. The results with both strains (LNH33 and LNH-ST) showed that glucose was completely consumed in the first stage, but xylose was partially converted (11.4 and $58.3 \%$ conversion, respectively) [49]. At the steady stage of the second stage xylose conversion reached a maximum of only $86.4 \%$, with an ethanol production of 13.8 and $21 \mathrm{~g} / \mathrm{L}$, respectively [49].

In this case, both strains were unable to convert the total concentration of xylose in the feed, even with the use of two-stages connected in series at a low dilution rate [49]. Another study also reported the co-fermentation of mixed sugars (glucose, $30 \mathrm{~g} / \mathrm{L}$; xylose, $15 \mathrm{~g} / \mathrm{L}$ ) with the recombinant $S$. cerevisiae strain $424 \mathrm{~A}$ (LNH-ST), without aeration, in MSCC on YPD complex medium, and with three reactors of different working volumes connected in series [50]. At the steady-state, all glucose and $37 \%$ of xylose $(5.6 \mathrm{~g} / \mathrm{L})$ were consumed in the first stage $\left(\mathrm{D}=0.05 \mathrm{~h}^{-1}\right)$; however, with the use of the other two stages, only a conversion of xylose of $69 \%$ was reached at the exit of the third stage $\left(D=0.05 \mathrm{~h}^{-1}\right)$ [50].

Lastly, a combination of Z. mobilis and Scheffersomyces stipitis strains were also used to evaluate the conversion of sugar mixtures in TSCC [51]. The hexose-fermenting bacterium $Z$. mobilis strain MTCC91 was inoculated in the first stage, with no aeration; and the pentose-fermenting yeast Scheffersomyces stipitis strain CBS6054 was inoculated in the second stage under micro-aerated conditions $(0.2 \mathrm{vvm})$. After testing different flow rates of complex medium supplemented with glucose, $80 \mathrm{~g} / \mathrm{L}$; and xylose, $40 \mathrm{~g} / \mathrm{L}$, it was found that the best condition was $\mathrm{D}_{1}=0.071 \mathrm{~h}^{-1}$ and $\mathrm{D}_{2}=0.048 \mathrm{~h}^{-1}$. At these dilution rates, the first stage allowed a glucose conversion of $81 \%$, with no xylose conversion; while in the second stage, the remaining glucose was completely consumed, but only $62.5 \%$ of xylose was utilized by the yeast. The overall ethanol production was $50 \mathrm{~g} / \mathrm{L}$, equivalent to a $\mathrm{Q}_{\mathrm{P}}$ of $1.56 \mathrm{~g} / \mathrm{L} \mathrm{h}$ [51]. The $\mathrm{Q}_{\mathrm{P}}$ reported in that study was like the value obtained by MS04 strain in this study. However, the xylose/glucose ratio used by MS04 was higher, and the experiments were carried out using mineral medium, instead of complex medium.

In the present study, it is shown that TSCC was superior to SSCC since the total consumption of the sugar mixture was achieved at higher dilution rates $\left(D_{1}=D_{2}=0.15 h^{-1}\right)$, in comparison to the required in SSCC $\left(D=0.05 \mathrm{~h}^{-1}\right)$. The use of this higher D in TSCC allowed the system to reach a higher ethanol concentration and productivity at a given value of $\mathrm{D}$ in the steady state (Table 2 ), by simultaneously consuming the total 
concentration of sugars and a partial consumption of acetate, which was required only in small amounts for biosynthetic pathways. It is important to mention that the deletion of the gene encoding the native alcohol dehydrogenase $(a d h E)$ in strain MS04 makes unfeasible to metabolize acetate to ethanol via acetyl-CoA.: i.e. acetate $\rightarrow$ acetyl-CoA $\rightarrow$ ethanol. Furthermore, the conversion of acetate to pyruvate or phosphoenolpyruvate, also via acetyl-CoA, and then to ethanol by the recombinant ethanol pathway from $Z$. mobilis is also unlikely as acetyl-CoA should be metabolized through the tricarboxylic acid cycle. However, this metabolic route does not operate as a cycle under the limiting oxygen conditions tested. Hence, it is improbable that consumed acetate contributes to the formation of ethanol in the strain used in this study. The configuration of TSCC was also more efficient than batch culture because it showed a higher productivity and simultaneous consumption of sugars, as well as an operation that can be maintained for a long time, eliminating the downtime required in batch and fed-batch cultures, and thus extending the productivity of the system. As mentioned before, MSCC presents the advantages of operating each stage independently, thus it allows the evaluation of a different number of stages, working volumes, flow rates, media composition and operating conditions in each stage. The unlimited possibilities of operation in this type of systems make MSCC very attractive at the laboratory and industrial level. Also, MSCC could be used at production level with hemicellulosic hydrolysates, because these syrups usually have low viscosity and low amounts of suspended solids, which make them easy to pump to feed large-scale continuous fermenters, and, as shown here, can be managed to reduce the carbon catabolite repression phenomena and perform the co-fermentation of mixed sugars to produce SGB with ethanologenic E. coli.

As described above, all xylose was not consumed in stage one or in SSCC at D $\geq 0.1 \mathrm{~h}^{-1}$, and all glucose is not consumed at $D_{1}=0.3 \mathrm{~h}^{-1}$. As previously shown [35], at oxygen transfer rates above $1.55 \mathrm{mmol} /(\mathrm{L} \mathrm{h})$ (corresponding to $\mathrm{k}_{\mathrm{L}} \mathrm{a}=7.2 \mathrm{~h}^{-1}$ and dissolved oxygen values of zero) the specific growth rates and cell mass increased in batch cultures. Owing to the characteristics of chemostats, these facts suggest that, under the conditions evaluated in this study, cells in SSCC and TSCC were not carbon growth limited, but were oxygen limited for growth in stage 1 . Higher $\mathrm{k}_{\mathrm{L}}$ a values to $7.2 \mathrm{~h}^{-1}$ were not tested because a value of $13.6 \mathrm{~h}^{-1}$ (i.e. $90 \%$ above the value used) in batch cultures [35] provoked an increase in growth rate and cell mass formation, but a significant decrease in ethanol titer and yield. As seen in Fig. 2, the second stage in the TSCC basically is a xylose-conversion chemostat to ethanol at $\mathrm{D}=0.10-0.20 \mathrm{~h}^{-1}$, but not at dilution rates above $0.2 \mathrm{~h}^{-1}$. The growth rate of strain MS04 in batch cultures using glucose-xylose mixtures and a $k_{\mathrm{L}}$ a of $7.2 \mathrm{~h}^{-1}$ is in the range of $0.46-0.47 \mathrm{~h}^{-1}$ [35; and this study]. However, on xylose this parameter is in the range of $0.20-0.25 \mathrm{~h}^{-1}$ (also at a $\mathrm{k}_{\mathrm{L}} \mathrm{a}=7.2 \mathrm{~h}^{-1}$; unpublished results). Hence, at $\mathrm{D} \geq 0.2 \mathrm{~h}^{-1}$ the steadystate cell concentration in stage 2 was lower in comparison to lower dilutions, and not all xylose was consumed, and the ethanol titer decreased (Fig. 2). Moreover, cells did not wash-out because they were supplied from stage 1. Taking together these facts, probably in the second stage remnant xylose is efficiently metabolized to ethanol because, in comparison to SSCC, more oxygen is supplied, and the dilution rate is below the $\mu$ of strain MS04 grown on xylose as carbon source.

\section{Conclusions}

The knowledge of used production kinetics and bioengineering techniques to maximize the simultaneous substrate utilization of media containing mixed sugars, and to increase the productivity of a specific metabolite, is of utmost importance in the conversion of lignocellulosic materials to biofuels. In this work, evolved E. coli strain MS04 showed a good performance in the conversion of xylose/glucose mixtures into ethanol, compared to other ethanologenic strains, when it grew in batch, single- and two-stage continuous cultures, achieving volumetric productivities higher than $1.5 \mathrm{~g} / \mathrm{L} \mathrm{h}$ in mineral media without antibiotics, and in the presence of inhibitors, such as acetate. It was demonstrated that the two-stage continuous culture is a better strategy than batch culture to co-ferment xylose/glucose mixtures to ethanol, since the ethanol productivity was higher in this system. In addition, for the conversion of higher concentration of xylose or mixed sugars, and the corresponding high ethanol productivities, the use of other configurations in continuous cultures, such as immobilization of the cells, or cell recycling (internal or external), could be a good alternative to enhance pentose sugars conversion, overall yield, and productivity of ethanol. The present work can be useful for further studies in continuous culture with recombinant ethanologenic strains, which could increase the simultaneous conversion of mixed sugars, thus eliminating or reducing the phenomenon of catabolite repression by glucose and enhancing the productivity of the system.

\section{Abbreviations}

adh: alcohol dehydrogenase gene; CCR: carbon catabolite repression; D: dilution rate; $D_{1}$ : dilution rate in the first-stage of two-stage continuous culture; $\mathrm{D}_{2}$ : dilution rate in the second-stage of two-stage continuous culture; $\mathrm{D}_{\mathrm{G}}$ : global dilution rate in the two-stage continuous culture; $\mathrm{DCW}$ : dry cell weight; EtOH: ethanol; F: flow of feed medium; $k_{L}$ a: volumetric oxygen transfer coefficient; FGB: first-generation bioethanol; G: glucose; MSCC: multi-stage continuous culture; mM: millimolar; $\mathrm{OD}_{600}$ : optical density at $600 \mathrm{~nm}$; pdc: 
pyruvate decarboxylase gene; Qp: volumetric ethanol productivity; qp: specific ethanol productivity; SGB: second-generation bioethanol; SSCC: single-stage continuous culture; TSCC: two-stage continuous culture; V: working volume; $X$ : xylose; $Y_{p s}$ : ethanol yield on sugars consumed as percentage of the maximum theoretical; $Y_{p x}$ : specific ethanol product yield; $Y_{X s}$ : cell yield on consumed sugars; $x_{1}$ : cell concentration at the steady-state of the first-stage of two-stage continuous culture; $\mathrm{x}_{2}$ : cell concentration at the steady-state of the secondstage of two-stage continuous culture; $\mu$ : specific growth rate; $\mu_{1}$ : specific growth rate in the first-stage of two-stage continuous culture; $\mu_{2}$ : specific growth rate in the second-stage of two-stage continuous culture; $\mu_{G}$ : global specific growth rate in the two-stage continuous culture; $\mu_{\max }$ : maximum specific growth rate; $\theta_{c}$ : liquid residence time.

\section{Acknowledgements}

We would like to thank, Georgina Hernández Chávez, Mercedes Enzaldo, Martín Patiño and Mario Trejo for technical assistance. We appreciate the valuable contributions of the anonymous reviewers that allowed us to improve this manuscript.

\section{Authors' contributions}

MTFS contributed to design, acquisition, and analysis of data, preparation of the manuscript and carried out the experiments and analysis. JGM contributed to the revision of the project and manuscript. FB and GG contributed to the revision of the manuscript; OTR contributed to the revision of the project and manuscript, and AM contributed to the concept and design of the investigation in addition to data analysis, preparation and revision of the manuscript. All authors read and approved the final manuscript.

\section{Funding}

This work was supported by DGAPA/PAPIIT/UNAM IT201414 and IV100119 and the Mexican Council of Science and Technology (CONACYT) FONCICYT ERANet-LAC Grant SMIBIO - C0013-248192. M.T.F-S. held a scholarship from CONACYT.

\section{Availability of data and materials}

All data generated or analyzed during this study are included in this published article.

\section{Ethics approval and consent to participate}

Not applicable.

\section{Consent for publication}

We state that all the authors (Marco T. Fernández-Sandoval, Juvencio GalíndezMayer, Francisco Bolívar, Guillermo Gosset, Octavio T. Ramírez and Alfredo Martinez) mutually agree that this research work should be submitted to Microbial Cell Factories; it is an original work of the above-mentioned authors; and that the article has not been published or submitted in any other peer-reviewed journal.

\section{Competing interests}

The authors declare that they have no competing interests.

\begin{abstract}
Author details
${ }^{1}$ Departamento de Ingeniería Celular y Biocatálisis, Instituto de Biotecnología, Universidad Nacional Autónoma de México, Av. Universidad 2001, Colonia Chamilpa, 62210 Cuernavaca, Morelos, Mexico. ${ }^{2}$ Laboratorio de Bioingeniería, Escuela Nacional de Ciencias Biológicas, Instituto Politécnico Nacional, Carpio y Plan de Ayala, Col. Santo Tomás, CP 11340 Mexico, D.F., Mexico. ${ }^{3}$ Departamento de Medicina Molecular y Bioprocesos, Instituto de Biotecnología, Universidad Nacional Autónoma de México, Av. Universidad 2001, Colonia Chamilpa, 62210 Cuernavaca, Morelos, Mexico.
\end{abstract}

Received: 11 March 2019 Accepted: 10 August 2019 Published online: 23 August 2019

\section{References}

1. Jönsson $\sqcup$, Alriksson B, Nilvebrant N. Bioconversion of lignocellulose: inhibitors and detoxification. Biotechnol Biofuels. 2013;6:16.
2. Förster AH, Gescher J. Metabolic engineering of Escherichia coli for production of mixed-acid fermentation end products. Front Bioeng Biotechnol. 2014;2:16.

3. Saini JK, Saini R, Tewari L. Lignocellulosic agriculture wastes as biomass feedstocks for second-generation bioethanol production: concepts and recent developments. 3 Biotech. 2015;5:337-53.

4. Carroll A, Somerville C. Cellulosic biofuels. Annu Rev Plant Biol. 2009;60:165-82.

5. Limayem A, Ricke SC. Lignocellulosic biomass for bioethanol production: current perspectives, potential issues and future prospects. Prog Energy Combust Sci. 2012;38:449-67.

6. Zanin GM, Santana CC, Bon EPS, Giordano RCL, De Moraes FF, Andrietta SR, De Carvalho Neto CC, Macedo IC, Larh Fo D, Ramos LP, Fontana JD. Brazilian bioethanol program. Appl Biochem Biotechnol. 2000;84-86:1147-61.

7. Wu Y, Shen X, Yuan Q, Yan Y. Metabolic engineering strategies for coutilization of carbon sources in microbes. Bioengineering. 2016;3(1):10.

8. Jordan DB, Bowman MJ, Braker JD, Dien BS, Hector RE, Lee CC, Mertens JA, Wagschal K. Plant cell walls to ethanol. Biochem J. 2012;442:241-52.

9. Jönsson LJ, Martín C. Pretreatment of lignocellulose: formation of inhibitory by-products and strategies for minimizing their effects. Biores Technol. 2016;199:103-12.

10. Munjal N, Mattam AJ, Pramanik D, Srivastava PS, Yazdani SS. Modulation of endogenous pathways enhances bioethanol yield and productivity in Escherichia coli. Microb Cell Fact. 2012;11:145.

11. Nieves $L M$, Panyon $L A$, Wang $X$. Engineering sugar utilization and microbial tolerance toward lignocellulose conversion. Front Bioeng Biotechnol. 2015;3:17.

12. Winters P. Current status of cellulosic biofuel commercialization in the United States. Ind Biotechnol. 2011;7:365-74.

13. Kim J, Block DE, Mills DA. Simultaneous consumption of pentose and hexose sugars: an optimal microbial phenotype for efficient fermentation of lignocellulosic biomass. Appl Microbiol Biotechnol. 2010;88:1077-85.

14. Saha BC. Hemicellulose bioconversion. J Ind Microbiol Biotechnol. 2003;30:279-91.

15. Bayrock DP, Ingledew WM. Ethanol production in multistage continuous, single stage continuous, Lactobacillus-contaminated continuous, and batch fermentations. World J Microbiol Biotechnol. 2005;21:83-8.

16. Nevoigt E. Progress in metabolic engineering of Saccharomyces cerevisiae. Microbiol Mol Biol Rev. 2008;72:379-412

17. Young E, Lee S-M, Alper $\mathrm{H}$. Optimizing pentose utilization in yeast: the need for novel tools and approaches. Biotechnol Biofuels. 2010;3:24.

18. Martin GJO, Knepper A, Zhou B, Pamment NB. Performance and stability of ethanologenic Escherichia coli strain FBR5 during continuous culture on xylose and glucose. J Ind Microbiol Biotechnol. 2006;33:834-44.

19. Ohta K, Beall DS, Mejia JP, Shanmugam KT, Ingram LO. Genetic improvement of Escherichia coli for ethanol production: chromosomal integration of Zymomonas mobilis genes encoding pyruvate decarboxylase and alcohol dehydrogenase II. Appl Environ Microbiol. 1991;57:893-900.

20. Yomano LP, York SW, Ingram LO. Isolation and characterization of ethanoltolerant mutants of Escherichia coli KO1 1 for fuel ethanol production. J Ind Microbiol Biotechnol. 1998;20:132-8.

21. Dien BS, Cotta MA, Jeffries TW. Bacteria engineered for fuel ethanol production: current status. Appl Microbiol Biotechnol. 2003;63:258-66.

22. Fernández-Sandoval MT, Huerta-Beristain G, Trujillo-Martinez B, Bustos $P$, Gonzalez V, Bolivar F, Gosset G, Martinez A. Laboratory metabolic evolution improves acetate tolerance and growth on acetate of ethanologenic Escherichia coli under non-aerated conditions in glucose-mineral medium. Appl Microbiol Biotechnol. 2012;96:1291-300.

23. Gao Q, Zhang M, McMillan JD, Kompala DS. Characterization of heterologous and native enzyme activity profiles in metabolically engineered Zymomonas mobilis strains during batch fermentation of glucose and xylose mixtures. Appl Biochem Biotechnol. 2002;98-100:341-55.

24. Ingram LO, Aldrich HC, Borges ACC, Causey TB, Martinez A, Morales F, Saleh A, Underwood SA, Yomano LP, York SW, Zaldivar J, Zhou S. Enteric bacterial catalyst for fuel ethanol production. Biotechnol Prog. 1999;15:855-66.

25. Vinuselvi P, Kim MK, Lee SK, Ghim CM. Rewiring carbon catabolite repression for microbial cell factory. BMB Rep. 2012;45(2):59-70. 
26. Klinke HB, Thomsen AB, Ahring BK. Inhibition of ethanol-producing yeast and bacteria by degradation products produced during pre-treatment of biomass. Appl Microbiol Biotechnol. 2004;66:10-26.

27. Görke B, Stülke J. Carbon catabolite repression in bacteria: many ways to make the most out of nutrients. Nat Rev Microbiol. 2008;6(8):613-24.

28. Nichols NN, Dien BS, Bothast RJ. Use of catabolite repression mutants for fermentation of sugar mixtures to ethanol. Appl Microbiol Biotechnol. 2001;56:120-5.

29. Chiang C, Lee HM, Guo HJ, Wang ZW, Lin L, Chao Y. Systematic approach to engineer Escherichia coli pathways for co-utilization of a glucose-xylose mixture. J Agric Food Chem. 2013;61:7583-90.

30. Gresham D, Dunham MJ. The enduring utility of continuous culturing in experimental evolution. Genomics. 2014;104(6):399-405.

31. Hoskisson PA, Hobbs G. Continuous culture-making a comeback? Microbiology. 2005;151:3153-9.

32. Brethauer $S$, Wyman CE. Review: continuous hydrolysis and fermentation for cellulosic ethanol production. Biores Technol. 2010;101:4862-74.

33. Zhou B, Martin GJO, Pamment NB. Increased phenotypic stability and ethanol tolerance of recombinant Escherichia coli KO11 when immobilized in continuous fluidized bed culture. Biotechnol Bioeng. 2008;100:627-33.

34. Bayrock DP, Ingledew WM. Application of multistage continuous fermentation for production of fuel alcohol by very-high-gravity fermentation technology. J Ind Microbiol Biotechnol. 2001;27:87-93.

35. Fernández-Sandoval MT, Galíndez-Mayer J, Moss-Acosta CL, Gosset G, Martinez A. Volumetric oxygen transfer coefficient as a means of improving volumetric ethanol productivity and a criterion for scaling up ethanol production with Escherichia coli. J Chem Tech Biotechnol. 2017;92:981-9.

36. Martinez A, Grabar TB, Shanmugam KT, Yomano LP, York SW, Ingram LO. Low salt medium for lactate and ethanol production by recombinant Escherichia coli B. Biotechnol Lett. 2007;29:397-404.

37. Castro E, Nieves IU, Mullinix MT, Sages WJ, Hoffman RW, FernándezSandoval MT, Tian Z, Rockwood DL, Tamang B, Ingram LO. Optimization of dilute-phosphoric-acid steam pretreatment of Eucalyptus benthamii for biofuel production. Appl Energy. 2014;125:76-83.

38. Castro E, Nieves IU, Rondón V, Sages WJ, Fernández-Sandoval MT, Yomano LP, York SW, Erickson J, Vermerris W. Potential for ethanol production from different sorghum cultivars. Ind Crops Prod. 2017;109:367-73.

39. Trinh CT, Unrean P, Srienc F. Minimal Escherichia coli cell for the most efficient production of ethanol from hexoses and pentoses. Appl Environ Microbiol. 2008;74(12):3634-43.

40. Dien BS, Nichols NN, O'Bryan PJ, Bothast RJ. Development of new ethanologenic Escherichia coli strains for fermentation of lignocellulosic biomass. Appl Biochem Biotechnol. 2000;84-86:181-96.
41. Madhavan A, Tamalampudi S, Srivastava A, Fukuda H, Bisaria VS, Kondo A. Alcoholic fermentation of xylose and mixed sugars using recombinant Saccharomyces cerevisiae engineered for xylose utilization. Appl Microbiol Biotechnol. 2009;82:1037-47.

42. Kuyper M, Toirkens MJ, Diderich JA, Winkler AA, van Dijken JP, Pronk JT. Evolutionary engineering of mixed-sugar utilization by a xylose-fermenting Saccharomyces cerevisiae strain. FEMS Yeast Res. 2005;5:925-34.

43. Zhou H, Cheng J, Wang BL, Fink GR, Stephanopoulos G. Xylose isomerase overexpression along with engineering of the pentose phosphate pathway and evolutionary engineering enable rapid xylose utilization and ethanol production by Saccharomyces cerevisiae. Metab Eng. 2012;14:611-22.

44. Hilaly AK, Karim MN, Linden JC. Comparison of ethanol production from xylose by a recombinant Escherichia coli in batch, fedbatch and continuous fermentations. J Gen Appl Microbiol. 1994;40:463-7.

45. Eliasson A, Christensson C, Wahlbom CF, Hahn-Hägerdal B. Anaerobic xylose fermentation by recombinant Saccharomyces cerevisiae carrying $X Y L 1, X Y L 2$, and XKS1 in mineral medium chemostat cultures. Appl Environ Microbiol. 2000;66(8):3381-6.

46. Unrean P, Srienc F. Continuous production of ethanol from hexoses and pentoses using immobilized mixed cultures of Escherichia coli strains. J Biotechnol. 2010;150:215-23.

47. Roca C, Olsson L. Increasing ethanol productivity during xylose fermentation by cell recycling of recombinant Saccharomyces cerevisiae. Appl Microbiol Biotechnol. 2003;60:560-3.

48. Lawford HG, Rousseau JD, Mohagheghi A, McMillan JD. Continuous cultures studies of xylose-fermenting Zymomonas mobilis. Appl Biochem Biotechnol. 1998;70-72:353-67.

49. Toon ST, Philippidis GP, Ho NWY, Chen Z, Brainard A, Lumpkin RE, Riley CJ. Enhanced cofermentation of glucose and xylose by recombinant Saccharomyces yeast strains in batch and continuous operating modes. Appl Biochem Biotechnol. 1997;63-65:243-55.

50. Govindaswamy S, Vane LM. Multi-stage continuous culture fermentation of glucose-xylose mixtures to fuel ethanol using genetically engineered Saccharomyces cerevisiae 424A. Biores Technol. 2010;101:1277-84.

51. Chaudhary G, Ghosh S. Two-reactor, continuous culture fermentation for fuel ethanol production from lignocellulosic acid hydrolysate using Zymomonas mobilis and Scheffersomyces stipitis. RDC Adv. 2014;4:36412-8.

\section{Publisher's Note}

Springer Nature remains neutral with regard to jurisdictional claims in published maps and institutional affiliations.

\footnotetext{
Ready to submit your research? Choose BMC and benefit from:

- fast, convenient online submission

- thorough peer review by experienced researchers in your field

- rapid publication on acceptance

- support for research data, including large and complex data types

- gold Open Access which fosters wider collaboration and increased citations

- maximum visibility for your research: over $100 \mathrm{M}$ website views per year
}

At BMC, research is always in progress.

Learn more biomedcentral.com/submissions 